\title{
Interferência da metodologia nos resultados de bioensaios de seleção de fungos entomopatogênicos para o controle de insetos
}

\author{
Patricia Helena Santoro(1), Pedro Manuel Oliveira Janeiro Neves ${ }^{(1)}$, Talita Moretto Alexandre ${ }^{(2)}$ \\ e Luís Francisco Angeli Alves ${ }^{(3)}$
}

\begin{abstract}
(1)Universidade Estadual de Londrina, Centro de Ciências Agrárias, Rod. Celso Garcia Cid, Campus Universitário, Caixa Postal 6001, CEP 86051-990 Londrina, PR. E-mail: ph_santoro@yahoo.com.br, pedroneves@uel.br (2)Universidade Federal do Paraná, Dep. de Zoologia, Caixa Postal 19020, CEP 81531-980 Curitiba, PR. E-mail: tma_br@yahoo.com.br (3)Universidade do Oeste do Paraná, Centro de Ciências Biológicas e da Saúde, R. Universitária 2.064, Caixa Postal 000711, CEP 85814-110 Cascavel, PR. E-mail: Ifaalves@unioeste.br
\end{abstract}

\begin{abstract}
Resumo - O objetivo deste trabalho foi avaliar a interferência dos métodos de bioensaios nos resultados de seleção de fungos entomopatogênicos, com a utilização de quatro isolados de Beauveria bassiana e adultos de Alphitobius diaperinus. Quanto à forma de inoculação, foram testados os seguintes métodos: imersão do inseto em suspensão de conídios; pulverização da suspensão sobre insetos; e tratamento de superfície com a suspensão em oito concentrações. Para avaliar a interferência do tempo de imersão sobre a mortalidade, os insetos foram colocados em suspensão de conídios por 1, 10, 20, 30, 40, 50 e 60 segundos. Conídios produzidos em arroz, insetos e meio sintético foram utilizados para avaliar a influência do substrato na virulência dos isolados. Os isolados UNIOESTE 4 e CG 152 foram os mais virulentos pelos métodos imersão e pulverização, respectivamente; em tratamento de superfície, a mortalidade foi baixa para todos os isolados. Observou-se que a mortalidade dos insetos é proporcional ao tempo em que ficam imersos, com tendência a se estabilizar após 40 segundos. Os isolados CG 71 e CG 152, produzidos sobre insetos, foram menos virulentos. Os métodos utilizados podem influenciar os resultados de bioensaios de seleção de fungos para o controle de insetos.
\end{abstract}

Termos para indexação: Beauveria bassiana, Alphitobius diaperinus, controle biológico, cascudinho, aviário, inoculação.

\section{Interference of bioassay methods on the results of entomopathogenic fungi selection for insect control}

\begin{abstract}
The objective of this work was to evaluate the interference of bioassay methods on the entomopathogenic fungi selection, using four Beauveria bassiana isolates and Alphitobius diaperinus adults. Regarding the inoculation form on insect mortality, the following methods were tested: insects immersion in a conidia suspension; spraying of a suspension over the insects; and surface treatment with eight concentrations. In order to evaluate the interference of immersion time on mortality, insects were immersed in a fungal suspension for $1,10,20,30,40,50$, and 60 seconds. Conidia produced on rice, insects, and synthetic medium were used to evaluate the influence of substrate on the virulence of the isolates. UNIOESTE 4 isolate and CG 152 were the most virulents for the methods immersion and spray, respectively; however, mortality was low in all isolates for the surface treatment. Mortality was proportional to the time during which insects stayed immersed, with a tendency to become stable after 40 seconds. Conidia from isolates CG 71 and CG 152 produced on insects were less virulent. The methods may influence bioassay results in the fungi selection for insects control.
\end{abstract}

Index terms: Beauveria bassiana, Alphitobius diaperinus, biologic control, lesser mealworm, poultry houses.

\section{Introdução}

A necessidade de reduzir os impactos ambientais, causados pelo uso excessivo de agrotóxicos, tem motivado estudos de formas alternativas para controle de pragas, entre elas, a utilização de fungos entomopatogênicos. Algumas espécies, como Beauveria bassiana (Bals.) e Metarhizium anisopliae (Metsch.) apresentam amplo espectro de hospedeiros e alta variabilidade genética (Alves, 1998) por isso é fundamental realizar bioensaios para selecionar isolados mais virulentos à praga-alvo.
Além da patogenicidade e virulência, outras características, como a resistência a condições ambientais adversas, crescimento vegetativo, produção de conídios in vitro, capacidade de disseminação de conídios e compatibilidade com insumos agropecuários, são desejáveis na seleção de um agente de controle microbiano (Charnley, 1997; Alves, 1998).

Em várias linhas de pesquisa, existem protocolos de métodos a serem seguidos, que possibilitam comparações entre os resultados obtidos em diferentes 
trabalhos. No entanto, quando utilizam fungos entomopatogênicos, os pesquisadores desenvolvem métodos de bioensaio e, ao comparar os resultados obtidos com os presentes na literatura, na maioria das vezes, não levam em consideração o procedimento metodológico utilizado.

Entre os fatores que variam nos testes para avaliar a ação dos fungos em insetos, estão: o modo de inoculação; o número de insetos utilizados; a viabilidade do patógeno; as concentrações de conídios; o período e modo de avaliação; e a mortalidade considerada (total, confirmada e corrigida). Essas variações podem influenciar os resultados obtidos, portanto as condições de bioensaios devem estar o mais próximo possível daquelas em que o patógeno será aplicado, assim o experimento possibilitará melhor previsão do que ocorrerá em condições de campo.

Os fungos infectam os insetos, preferencialmente, pela superfície do tegumento (Boucias \& Pendland, 1998). Por isso, os métodos de inoculação mais utilizados são: pulverização sobre o inseto (Neves \& Hirose, 2005; Rohde et al., 2006), imersão do inseto na suspensão (Loureiro \& Monteiro, 2005); e tratamento da superfície com a suspensão de conídios (Batista Filho et al., 1992).

Variações também podem ocorrer dentro de um mesmo método de inoculação, como o de imersão, em que Alves et al. (1985) imergiram larvas de Diatraea saccharalis (Fabr.) por 4 segundos e González García et al. (1993) imergiram Hypothenemus hampei (Ferrari) por 2 minutos.

A concentração da suspensão do fungo deve ser preparada com base no número de conídios viáveis, para isso é necessária a realização prévia de testes de viabilidade. Além disso, recomenda-se reativar a capacidade infectiva dos isolados, por meio da inoculação em insetos (Velez et al., 1997), e utilizar conídios de mesma repicagem, pois alguns autores afirmam que, após vários repiques em meio artificial, a capacidade infectiva dos conídios tende a diminuir (Ferron et al., 1972, citados por Ignoffo et al., 1982).

Outro aspecto importante na seleção de isolados é a determinação do período de avaliação, a qual deve se basear na ação do fungo para a praga-alvo, e nos objetivos a serem alcançados, isto é, maior mortalidade em curto período de tempo ou maior mortalidade em períodos mais prolongados.

Este trabalho teve como objetivo estudar a interferência dos métodos de bioensaios nos resultados obtidos em experimentos que avaliam a ação de fungos entomopatogênicos aos insetos, na seleção dos isolados mais virulentos, tendo como modelo o fungo Beauveria bassiana e o inseto Alphitobius diaperinus.

\section{Material e Métodos}

Utilizaram-se os seguintes isolados dos fungos Beauveria bassiana: CG 71, CG 152, UNIOESTE 4 e UNIOESTE 40, que se mostraram virulentos aos adultos de Alphitobius diaperinus em testes preliminares. Os isolados se encontram armazenados no Banco de Entomopatógenos, do Laboratório de Controle Microbiano de Insetos, da Universidade Estadual de Londrina, PR. Para recuperar a capacidade infectiva, os conídios foram inoculados em adultos de A. diaperinus, posteriormente reisolados dos insetos, $\mathrm{e}$ multiplicados por duas vezes em placas de Petri com meio BDA. As placas foram mantidas em câmara climatizada $\left(25 \pm 1^{\circ} \mathrm{C}\right.$ e fotoperíodo de 12 horas) por 10 dias. Foram utilizados, em todos os experimentos, conídios de segunda repicagem com viabilidade acima de $95 \%$.

Os insetos utilizados no experimento foram adultos da espécie A. diaperinus, em razão da facilidade de serem encontrados em grandes quantidades. No dia anterior à montagem dos experimentos, os insetos foram coletados em aviário comercial, localizado no Município de Londrina. Em laboratório, foram separados da "cama", alimentados com ração para aves e mantidos em condição ambiente.

Para avaliar a interferência do modo de inoculação do fungo sobre o inseto, foram testados os métodos de imersão, pulverização e tratamento de superfície. Para estimar a concentração letal $\left(\mathrm{CL}_{50}\right)$, em cada método, foram utilizadas oito concentrações de conídios e uma testemunha que continha apenas solução aquosa de Tween 20 a $0,02 \%$ (v/v) esterilizada. A definição das concentrações a serem utilizadas foi feita com base em pré-testes, a fim de se determinar uma concentração que matasse cerca de $10 \%$, e outra que matasse $90 \%$ da população, com as outras concentrações utilizadas distribuídas entre esses extremos (Robertson et al., 1984).

Para o método de imersão, os conídios foram adicionados à solução aquosa de Tween 20 a 0,02\% (v/v) esterilizada e as suspensões foram padronizadas nas concentrações de $5 \times 10^{4}, 1 \times 10^{5}, 5 \times 10^{5}, 1 \times 10^{6}, 5 \times 10^{6}, 1 \times 10^{7}$, $5 \times 10^{7}$ e $1 \times 10^{8}$ conídios $\mathrm{mL}^{-1}$; a testemunha continha apenas o veículo da suspensão. Grupos de 50 insetos 
foram imersos em $5 \mathrm{~mL}$ de cada suspensão, por 30 segundos.

Na pulverização de conídios sobre o inseto, as concentrações das suspensões utilizadas foram de $5 \times 10^{5}$, $1 \times 10^{6}, 5 \times 10^{6}, 1 \times 10^{7}, 5 \times 10^{7}, 1 \times 10^{8}, 5 \times 10^{8}$ e $1 \times 10^{9}$ conídios $\mathrm{mL}^{-1}$ e testemunha. Com um pulverizador (Airbrush acoplado a um compressor/aspirador Fanen-Diapump), 0,5 mL da suspensão foi pulverizado sobre 50 insetos acondicionados em placas de Petri, onde permaneceram durante todo o período de avaliação.

No tratamento de superfície, pelo fato de não ter sido possível, em razão da baixa mortalidade observada nos pré-testes, estabelecer uma concentração que matasse cerca de $90 \%$ dos insetos, optou-se por utilizar as mesmas concentrações do método de pulverização e uma testemunha. Foi pulverizado, uniformemente, sobre placas de Petri ( $9 \mathrm{~cm}$ de diâmetro), 0,5 mL das suspensões, e as placas foram mantidas semi-abertas, à temperatura ambiente, por cerca de $30 \mathrm{~min}$ para secagem completa. Grupos de 50 insetos foram colocados nas placas, onde permaneceram durante o período de avaliação.

Com o objetivo de se verificar a influência do tempo de imersão dos insetos em suspensão do fungo, grupos de 50 insetos foram colocados em $5 \mathrm{~mL}$ de uma suspensão com $8 \times 10^{5}$ conídios $\mathrm{mL}^{-1}$ do isolado UNIOESTE $4\left(\mathrm{CL}_{50}\right.$ estimada para o método de imersão), por 1, 10, 20, 30, 50 e 60 segundos e, em seguida, acondicionados em placa de Petri $(9 \mathrm{~cm} \mathrm{de}$ diâmetro).

Para observar a influência do substrato de produção dos conídios sobre a virulência do fungo, utilizaram-se os isolados CG 71, CG 152, UNIOESTE 4 e UNIOESTE 40, produzidos em meio sintético para geração de esporos de Beauveria spp. (Alves et al., 1998), arroz pré-cozido e o próprio inseto. Os conídios foram repicados em placas de Petri, com meio sintético para produção de esporos de Beauveria spp., e foram mantidos em câmara climatizada $\left(25 \pm 1^{\circ} \mathrm{C}\right.$ e fotoperíodo de 12 horas) por 10 dias. Em arroz, os conídios foram produzidos conforme Leite et al. (2003) e acondicionados sob as mesmas condições já mencionadas. Para obtenção dos conídios produzidos sobre os insetos, estes foram pulverizados com suspensão de conídios e, após sua morte, foram acondicionados em câmara úmida $\left(25 \pm 1^{\circ} \mathrm{C}\right.$ e fotoperíodo de 12 horas) por 10 dias.

Para preparar as suspensões, os conídios foram retirados do meio sintético com o auxílio de uma espátula; o arroz e os insetos foram colocados em solução aquosa de Tween 20 a 0,02\% (v/v) esterilizada, e foram agitados para desprendimento dos conídios. As suspensões foram padronizadas na concentração de $8 \times 10^{5}$ conídios $\mathrm{mL}^{-1}\left(\mathrm{CL}_{50}\right.$ do isolado UNIOESTE 4 para o método de imersão), e os insetos pelo método de imersão descrito no primeiro experimento.

Em todos os experimentos, os insetos foram acondicionados em placas de Petri ( $9 \mathrm{~cm}$ diâmetro), mantidos em câmara climatizada $\left(25 \pm 1^{\circ} \mathrm{C}\right.$ e fotoperíodo de 12 horas) e alimentados com ração esterilizada para aves. As avaliações foram realizadas diariamente, por 10 dias. Os insetos mortos foram lavados em solução de hipoclorito de sódio (1\%) e acondicionados em câmara úmida $\left(25 \pm 1^{\circ} \mathrm{C}\right.$ e fotoperíodo de 12 horas), para produção de conídios sobre o corpo e confirmação da mortalidade.

Durante a realização dos bioensaios, foram observados e descritos os sintomas de infecção do fungo nos insetos.

O delineamento experimental foi inteiramente casualizado, com cinco repetições de 50 insetos para cada tratamento, número recomendado por Robertson et al. (1984). Somente no estudo da influência do tempo de imersão foram utilizadas seis repetições com o mesmo número de insetos. Os dados de mortalidade foram corrigidos em relação à testemunha, pela fórmula de Shneider e Orelli (Nakano et al., 1981). Na comparação entre métodos de inoculação, as estimativas das concentrações letais foram determinadas por Probit, pelo programa PoloPlus 1.0 (LeOra Software, 2003). Utilizou-se o teste qui-quadrado $\left(\chi^{2}\right)$, a $5 \%$ de probabilidade, para determinar a adequação dos dados ao modelo de Probit (Haddad, 1998). Nos demais testes, os dados foram submetidos à análise de variância, e as médias foram comparadas pelo teste de Tukey, a 5\% de probabilidade.

\section{Resultados e Discussão}

Ao se compararem os dados de mortalidade, confirmada e corrigida de A. diaperinus, provocada pelos diferentes isolados em cada método de inoculação, verificou-se que o método pode influenciar a seleção de isolados. O isolado UNIOESTE 4 foi o mais virulento para imersão, e, com exceção da concentração mais baixa $\left(5 \times 10^{5}\right.$ conídios $\left.\mathrm{mL}^{-1}\right)$, o CG 152 foi o mais virulento para pulverização (Tabelas 1 e 2). No tratamento de superfície, a mortalidade confirmada corrigida foi baixa para todos os isolados, que não diferiram estatisticamente entre si (Tabela 1). 
O método de imersão causou a maior mortalidade confirmada corrigida mas não diferiu, em algumas concentrações, do método de pulverização para os isolados CG 152 e UNIOESTE 40. Em tratamento de superfície, mesmo tendo-se utilizado as mesmas concentrações de conídios e quantidade de suspensão do método de pulverização, a mortalidade foi sempre inferior (Tabela 1). Essa diferença na mortalidade pode ser conseqüência do número de conídios que efetivamente en- traram em contato com o inseto, que no tratamento de superfície é, provavelmente, inferior, por ficarem os conídios restritos a algumas regiões do corpo, como pernas e parte ventral. Em pulverização e imersão, um maior número de conídios entra em contato com o corpo inteiro do inseto, com possibilidade de germinação e penetração, o que aumenta a mortalidade e diminui a $\mathrm{CL}_{50}$.

Ao comparar diferentes métodos de inoculação de B. bassiana em H. hampei, Fernandes et al. (1985)

Tabela 1. Mortalidade confirmada e corrigida (\%) de adultos de Alphitobius diaperinus, 10 dias após a inoculação, por diferentes isolados de Beauveria bassiana em conseqüência dos métodos de inoculação e concentrações ${ }^{(1)}$.

\begin{tabular}{|c|c|c|c|c|}
\hline Concentração (conídios $\mathrm{mL}^{-1}$ ) & Isolado & Imersão & Pulverização & Tratamento de superfície \\
\hline \multirow[t]{4}{*}{$5 \times 10^{5}$} & CG 71 & $12,80 \pm 1,32 \mathrm{Ab}$ & $5,20 \pm 0,58 \mathrm{Bb}$ & $0,40 \pm 0,24 \mathrm{Ca}$ \\
\hline & CG 152 & $17,60 \pm 1,33 \mathrm{Aa}$ & $13,00 \pm 1,14 \mathrm{Ba}$ & $0,00 \pm 0,00 \mathrm{Ca}$ \\
\hline & UNIOESTE 4 & $12,60 \pm 1,03 \mathrm{Ab}$ & $2,80 \pm 0,92 \mathrm{Bbc}$ & $0,00 \pm 0,00 \mathrm{Ca}$ \\
\hline & UNIOESTE 40 & $0,60 \pm 0,24 \mathrm{Ac}$ & $1,00 \pm 0,45 \mathrm{Ac}$ & $0,00 \pm 0,00 \mathrm{Aa}$ \\
\hline \multirow[t]{4}{*}{$1 \times 10^{6}$} & CG 71 & $15,80 \pm 0,73 \mathrm{Ac}$ & $7,80 \pm 0,58 \mathrm{Bc}$ & $0,60 \pm 0,24 \mathrm{Ca}$ \\
\hline & CG 152 & $20,00 \pm 1,58 \mathrm{Ab}$ & $20,40 \pm 1,63 \mathrm{Aa}$ & $0,20 \pm 0,20 \mathrm{Ba}$ \\
\hline & UNIOESTE 4 & $26,60 \pm 1,08 \mathrm{Aa}$ & $16,20 \pm 0,80 \mathrm{Bb}$ & $0,20 \pm 0,20 \mathrm{Ca}$ \\
\hline & UNIOESTE 40 & $2,00 \pm 0,71 \mathrm{Ad}$ & $1,60 \pm 0,40 \mathrm{Ad}$ & $0,00 \pm 0,00 \mathrm{Aa}$ \\
\hline \multirow[t]{4}{*}{$5 \times 10^{6}$} & CG 71 & $22,60 \pm 0,81 \mathrm{Ab}$ & $13,60 \pm 0,93 \mathrm{Bc}$ & $1,00 \pm 0,32 \mathrm{Ca}$ \\
\hline & CG 152 & $24,20 \pm 1,32 \mathrm{Bb}$ & $30,60 \pm 0,81 \mathrm{Aa}$ & $0,20 \pm 0,20 \mathrm{Ca}$ \\
\hline & UNIOESTE 4 & $43,60 \pm 1,72 \mathrm{Aa}$ & $22,80 \pm 1,32 \mathrm{Bb}$ & $0,00 \pm 0,00 \mathrm{Ca}$ \\
\hline & UNIOESTE 40 & $3,60 \pm 0,60 \mathrm{Ac}$ & $3,60 \pm 0,51 \mathrm{Ad}$ & $0,20 \pm 0,20 \mathrm{Ba}$ \\
\hline \multirow[t]{4}{*}{$1 \times 10^{7}$} & CG 71 & $31,60 \pm 0,68 \mathrm{Ab}$ & $18,60 \pm 0,75 \mathrm{Bc}$ & $1,40 \pm 024 \mathrm{Ca}$ \\
\hline & CG 152 & $32,60 \pm 0,60 \mathrm{Ab}$ & $32,40 \pm 1,72 \mathrm{Aa}$ & $0,40 \pm 0,24 \mathrm{Ba}$ \\
\hline & UNIOESTE 4 & $45,60 \pm 1,94 \mathrm{Aa}$ & $25,40 \pm 0,81 \mathrm{Bb}$ & $0,20 \pm 0,20 \mathrm{Ca}$ \\
\hline & UNIOESTE 40 & $8,60 \pm 0,51 \mathrm{Ac}$ & $6,60 \pm 0,81 \mathrm{Ad}$ & $0,40 \pm 0,24 \mathrm{Ba}$ \\
\hline \multirow[t]{4}{*}{$5 \times 10^{7}$} & CG 71 & $35,20 \pm 1,24 \mathrm{Ac}$ & $24,60 \pm 0,68 \mathrm{Bc}$ & $1,80 \pm 0,37 \mathrm{Ca}$ \\
\hline & CG 152 & $40,20 \pm 0,86 \mathrm{Ab}$ & $37,60 \pm 1,33 \mathrm{Aa}$ & $0,60 \pm 0,24 \mathrm{Ca}$ \\
\hline & UNIOESTE 4 & $47,40 \pm 1,21 \mathrm{Aa}$ & $34,00 \pm 1,14 \mathrm{Bb}$ & $2,80 \pm 0,86 \mathrm{Ca}$ \\
\hline & UNIOESTE 40 & $11,60 \pm 0,75 \mathrm{Ad}$ & $13,40 \pm 0,68 \mathrm{Ad}$ & $1,00 \pm 0,45 \mathrm{Ba}$ \\
\hline \multirow[t]{4}{*}{$1 \times 10^{8}$} & CG 71 & $41,40 \pm 1,17 \mathrm{Ab}$ & $29,20 \pm 1,83 \mathrm{Bb}$ & $3,20 \pm 0,73 \mathrm{Ca}$ \\
\hline & CG 152 & $44,20 \pm 0,49 \mathrm{Ab}$ & $42,20 \pm 2,22 \mathrm{Aa}$ & $3,60 \pm 0,24 \mathrm{Ba}$ \\
\hline & UNIOESTE 4 & $49,80 \pm 0,20 \mathrm{Aa}$ & $41,60 \pm 3,11 \mathrm{Ba}$ & $3,00 \pm 1,18 \mathrm{Ca}$ \\
\hline & UNIOESTE 40 & $21,40 \pm 0,75 \mathrm{Ac}$ & $21,40 \pm 0,40 \mathrm{Ac}$ & $1,40 \pm 0,51 \mathrm{Ba}$ \\
\hline
\end{tabular}

(1)Médias (土erro-padrão) seguidas por letras iguais, maiúsculas na linha e minúsculas na coluna, em uma mesma concentração, não diferem entre si pelo teste de Tukey, a $5 \%$ de probabilidade.

Tabela 2. Concentração letal cinqüenta $\left(\mathrm{CL}_{50}\right)$ de diferentes isolados de Beauveria bassiana, para adultos de Alphitobius diaperinus, por diferentes métodos de inoculação, 10 dias após a inoculação.

\begin{tabular}{|c|c|c|c|c|c|}
\hline \multirow[t]{2}{*}{ Isolado } & \multirow[t]{2}{*}{$\begin{array}{l}\text { Equação de } \\
\text { regressão }\end{array}$} & \multirow[t]{2}{*}{$\begin{array}{c}\text { CL } 50 \\
\left(10^{6} \text { conídios } \mathrm{mL}^{-1}\right)\end{array}$} & \multicolumn{2}{|c|}{$\begin{array}{c}\text { Intervalo de } \\
\text { confiança }(95 \%)\end{array}$} & \multirow[t]{2}{*}{$\chi^{2}$} \\
\hline & & & Inferior & Superior & \\
\hline \multicolumn{6}{|c|}{ Imersão } \\
\hline CG 71 & $Y=4,9839+0,7425 \times \log x$ & 5,69 & 3,88 & 8,60 & 15,08 \\
\hline CG 152 & $Y=6,2183+0,5962 \times \log x$ & 2,19 & 1,34 & 3,62 & 17,35 \\
\hline UNIOESTE 4 & $Y=3,5651+1,0885 \times \log x$ & 0,81 & 0,46 & 1,41 & 50,11 \\
\hline UNIOESTE 40 & $\mathrm{Y}=3,4244+0,7844 \times \log \mathrm{x}$ & 241,21 & 123,08 & 672,11 & 13,40 \\
\hline \multicolumn{6}{|c|}{ Pulverização } \\
\hline CG 71 & $Y=5,1842+0,4749 \times \log x$ & 70,57 & 54,49 & 93,07 & $4,33^{*}$ \\
\hline CG 152 & $Y=5,7638+0,6542 \times \log x$ & 2,92 & 1,68 & 4,64 & 14,72 \\
\hline UNIOESTE 4 & $Y=3,9875+0,8680 \times \log x$ & 8,44 & 4,42 & 15,12 & 43,62 \\
\hline UNIOESTE 40 & $Y=2,6908+0,8860 \times \log x$ & 177,42 & 146,92 & 217,09 & $5,69 *$ \\
\hline \multicolumn{6}{|c|}{ Tratamento de superfície } \\
\hline CG 71 & $Y=-1,2782+0,6197 \times \log x$ & - & - & - & 12,60 \\
\hline CG 152 & $Y=-2,7489+0,7688 \times \log x$ & - & - & - & $9,16^{*}$ \\
\hline UNIOESTE 4 & $Y=-4,1851+0,9627 \times \log x$ & - & - & - & 16,54 \\
\hline UNIOESTE 40 & $Y=-3,1370+0,7938 \times \log x$ & - & - & - & $1,51 *$ \\
\hline
\end{tabular}

*Não-significativo pelo teste do qui-quadrado, a $5 \%$ de probabilidade. 
observaram que a mortalidade confirmada foi maior quando os insetos foram colocados sobre folhas e grãos de café previamente tratados com suspensão de conídios, com 85 e $92 \%$ respectivamente, e baixa mortalidade na testemunha (16 e 6\%, respectivamente). Quando os insetos foram imersos em suspensão de conídios a mortalidade confirmada foi de $52 \%$, a mortalidade total foi de $100 \%$ e, na testemunha, foi de $66 \%$. Esses dados mostram que, provavelmente, a alta mortalidade total observada no método de imersão não foi provocada pelo fungo, mas sim pelo método utilizado. Esses resultados confirmam que o modo de inoculação influencia nos resultados obtidos em bioensaios.

Ao estudar o efeito do modo de exposição de Leptinotarsa decemlineata (Say) ao fungo B. bassiana, Fernandez et al. (2001) verificaram que para larvas pulverizadas com suspensão de conídios a mortalidade foi de $76 \%$, e quando as larvas foram colocadas em folhas pulverizadas com B. bassiana a mortalidade foi de $34 \%$, o que também corrobora os dados obtidos neste trabalho.

O conhecimento da concentração letal conduz a conclusões a respeito da potência dos patógenos, da sensi-
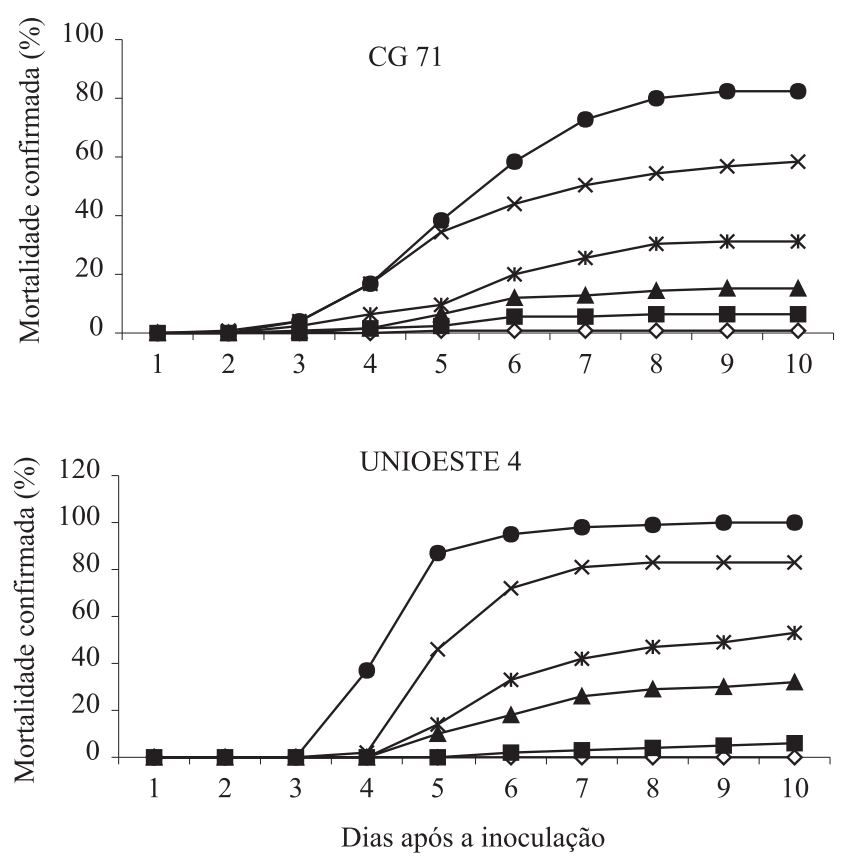

bilidade dos insetos à doença e até mesmo sobre previsões de controle de pragas, por meio dos inseticidas microbianos (Haddad, 1998). Pelo método de imersão, a menor $\mathrm{CL}_{50}$ foi do isolado UNIOESTE $4 \mathrm{e}$, pela pulverização, a menor $\mathrm{CL}_{50}$ foi a do isolado $C G$ 152, o que evidencia a interferência do método de inoculação na seleção de isolados mais virulentos. Pela análise de Probit, não foi possível estimar as $\mathrm{CL}_{50}$ para tratamento de superfície, em razão da baixa mortalidade (Tabela 2).

Analisando-se a distribuição de mortalidade de adultos de A. diaperinus pelos isolados de B. bassiana, foi possível verificar que, de modo geral, os insetos começaram a morrer a partir do segundo e terceiro dias após o contato com o inóculo. Os picos de mortalidade ocorreram entre o quarto, quinto e sexto dias, com tendência a se estabilizar a partir do sétimo e oitavo dias. Entretanto, para o isolado UNIOESTE 40, a mortalidade confirmada foi praticamente nula até o sexto dia após a inoculação, o que mostra um comportamento diferenciado (Figura 1).

Essa distribuição da mortalidade no decorrer do tempo pode variar conforme a espécie estudada, como no
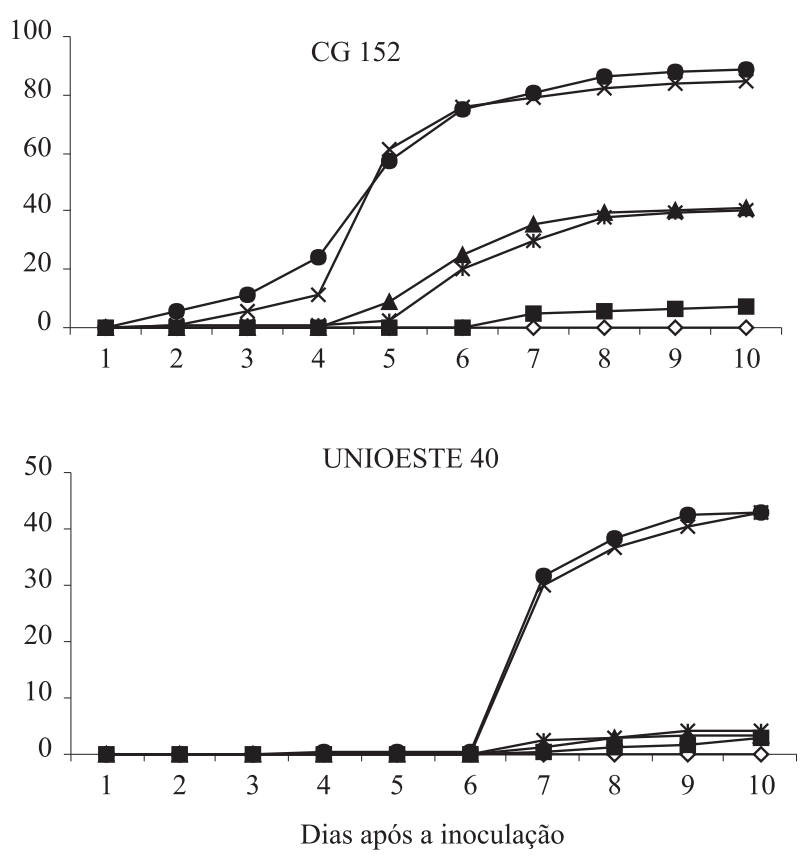

Figura 1. Mortalidade confirmada corrigida acumulada no decorrer do tempo de adultos de Alphitobius diaperinus por isolados de Beauveria bassiana, em diferentes métodos de inoculação e concentrações. * e **Concentração de $1 \times 10^{6}$ e $1 \times 10^{8}$ conídios $\mathrm{mL}^{-1}$, respectivamente. 
trabalho de Andalo et al. (2004), que observaram que para a cochonilha Dysmicoccus texensis (Tinsley), o início da mortalidade por $B$. bassiana ocorreu no sexto dia e estabilizou-se no décimo. Os autores explicaram que a presença de uma secreção pulverulenta sobre o inseto pode ter dificultado a penetração do fungo, o que retardou a mortalidade. Para Atta sexdens sexdens (Linnaeus), a mortalidade por B. bassiana ocorreu a partir do segundo dia e cessou ao sétimo dia após a inoculação (Loureiro \& Monteiro, 2005).

Com base nos resultados obtidos neste trabalho, verifica-se a necessidade da realização de um pré-teste que observe os períodos de início e término da mortalidade para o inseto e patógeno em estudo, afim de que não se subestime o potencial de controle para pragas que apresentam mortalidade mais tardia. Além disso, a distribuição da mortalidade, no decorrer do tempo, é um importante fator a ser considerado durante a seleção de isolados, tendo-se sempre como base os objetivos a serem atingidos. Por exemplo, para pragas que são vetores de doenças ou que causam danos severos em curto período de tempo, é necessário selecionar patógenos que provoquem alta mortalidade em menor período. Para pragas menos agressivas ou para culturas que suportem maiores danos, pode-se optar por um isolado que, além de virulento, seja mais persistente às condições adversas do ambiente e provoque mortalidade por períodos mais prolongados.

Ao se compararem os diferentes tempos de imersão, observou-se que a mortalidade confirmada aumentou em proporção direta ao tempo de imersão, com tendência a se estabilizar após 40 segundos (Tabela 3 ). A exposição de insetos por meio do método de imersão é normalmente utilizada em testes laboratoriais, no entanto, não existe um padrão quanto ao tempo em que os insetos ficam imersos, com grande variação, como por exemplo, 4 segundos para $D$. saccharalis (Alves et al.,

Tabela 3. Mortalidade confirmada e corrigida, em porcentagem de adultos de Alphitobius diaperinus submetidos a diferentes tempos de imersão em suspensão de conídios $\left(8 \times 10^{5}\right.$ conídios $\left.\mathrm{mL}^{-1}\right)$ do isolado UNIOESTE 4 de Beauveria bassiana, após 10 dias de avaliação ${ }^{(1)}$.

\begin{tabular}{cc}
\hline Tempo (segundos) & Mortalidade confirmada \\
\hline 60 & $35,66 \pm 2,27 \mathrm{a}$ \\
50 & $32,83 \pm 0,83 \mathrm{ab}$ \\
40 & $29,33 \pm 0,88 \mathrm{~b}$ \\
30 & $23,66 \pm 0,92 \mathrm{c}$ \\
20 & $18,00 \pm 0,73 \mathrm{~d}$ \\
10 & $12,83 \pm 0,87 \mathrm{e}$ \\
1 & $6,16 \pm 0,65 \mathrm{f}$ \\
\hline
\end{tabular}

${ }^{(1)}$ Médias ( \pm erro-padrão) seguidas por letras iguais não diferem entre si pelo teste de Tukey, a $5 \%$ de probabilidade.
1985) e até 2 minutos para H. hampei (González García et al., 1993). Desta maneira, considerando-se que o tempo de imersão influencia na mortalidade, existe a necessidade de se determinar previamente o tempo em que os insetos ficam imersos, e optar por menores períodos, afim de não se superestimar o potencial do patógeno, já que existe tendência de diminuição da $\mathrm{CL}_{50}$ à medida que é aumentado o tempo de imersão.

Observou-se a influência do substrato de produção de conídios sobre a virulência do fungo e verificou-se que conídios dos isolados CG 71 e CG 152, produzidos em insetos, foram menos virulentos que os produzidos em arroz e meio sintético (Tabela 4). Por meio da análise dos demais resultados, não foi possível estabelecer qual o melhor substrato, pois cada isolado se comportou de maneira distinta, possivelmente em razão da variabilidade genética entre eles, o que pode ter proporcionado um melhor ou pior aproveitamento dos nutrientes em determinado meio, provocando, assim, diferenças na virulência para os conídios produzidos em diferentes substratos. Entretanto, este estudo mostra que existe diferença na virulência entre conídios produzidos em diferentes meios, por isso, é importante utilizar, nos testes de seleção, o mesmo meio de cultura que será utilizado para produzir o fungo em larga escala para sua aplicação em campo. Hallsworth \& Magan $(1994,1995)$, citados por Burges (1998), observaram que a redução de glicerol, eritritol ou trialose produziram conídios de M. anisopliae, B. bassiana e Paecilomyces farinosus (Wise) Brown \& Smith mais virulentos, o que confirma a relação existente entre o meio de produção e a virulência.

Nas observações realizadas durante os experimentos, foi possível detectar os principais sintomas de infecção causados pelo fungo B. bassiana aos adultos de A. diaperinus. Entre os sintomas, se observou a diminuição na movimentação dos insetos infectados, em comparação aos insetos sadios. No dia antecedente à morte, os insetos apresentaram-se com a face ventral voltada para cima e as pernas afastadas do corpo, com

Tabela 4. Mortalidade confirmada e corrigida, em porcentagem de adultos de Alphitobius diaperinus, por diferentes isolados de Beauveria bassiana $\left(8 \times 10^{5}\right.$ conídios $\left.\mathrm{mL}^{-1}\right)$, produzidos em diferentes substratos ${ }^{(1)}$.

\begin{tabular}{lrrr}
\hline Isolado & \multicolumn{3}{c}{ Substrato } \\
\cline { 2 - 4 } & Arroz & Meio sintético & \multicolumn{1}{c}{ Inseto } \\
\hline CG 071 & $23,20 \pm 0,80 \mathrm{Cb}$ & $32,40 \pm 0,86 \mathrm{Ba}$ & $20,20 \pm 0,45 \mathrm{Bc}$ \\
CG 152 & $32,60 \pm 0,93 \mathrm{Ba}$ & $16,2 \pm 0,32 \mathrm{Db}$ & $6,20 \pm 0,97 \mathrm{Cc}$ \\
UNI 004 & $38,80 \pm 0,80 \mathrm{Aa}$ & $20,2 \pm 0,97 \mathrm{Cb}$ & $19,00 \pm 1,02 \mathrm{Bb}$ \\
UNI 040 & $31,20 \pm 1,21 \mathrm{Bb}$ & $41,8 \pm 0,58 \mathrm{Aa}$ & $29,40 \pm 0,51 \mathrm{Ab}$ \\
\hline
\end{tabular}

${ }^{(1)}$ Médias ( \pm erro-padrão) seguidas por letras iguais, maiúsculas na coluna e minúsculas na linha, não diferem entre si pelo teste de Tukey, a $5 \%$ de probabilidade. 
movimentos lentos quando tocados. Ao morrerem, apresentaram aspecto rígido e seco, pernas afastadas do corpo e o tegumento com coloração mais clara, quando comparado a insetos saudáveis. A exteriorização do patógeno sobre o corpo do inseto iniciou-se pelas suturas, dois dias após o acondicionamento em câmara úmida, e entre o 4 o e 5을 dias o corpo do inseto estava recoberto, quase totalmente, pelo fungo.

\section{Conclusões}

1. Os métodos utilizados nos bioensaios interferem nos resultados da seleção de isolados de fungos entomopatogênicos.

2. O tempo de imersão na suspensão de conídios de Beauveria bassiana aumenta a mortalidade confirmada de Alphitobius diaperinus, de forma diretamente proporcional, com estabilização após 40 segundos.

3. O período ideal para avaliação dos bioensaios de seleção de isolados de B. bassiana, para adultos de A. diaperinus, é de 10 dias.

4. O substrato utilizado para a produção do fungo interfere na virulência do patógeno.

\section{Referências}

ALVES, S.B. Fungos entomopatogênicos. In: ALVES, S.B. Controle microbiano de insetos. Piracicaba: Fealq, 1998. p.289-381.

ALVES, S.B.; ALMEIDA, J.E.M.; MOINO, A.; ALVES, L.F.A. Técnicas de laboratório. In: ALVES, S.B. Controle microbiano de insetos. Piracicaba: Fealq, 1998. p.637-711.

ALVES, S.B.; PÁDUA, L.E.M.; AZEVEDO, E.M.V.M.; ALMEIDA, L.C. Controle da broca da cana-de-açúcar pelo uso de Beauveria bassiana. Pesquisa Agropecuária Brasileira, v.20, p.403-406, 1985.

ANDALÓ, V.; MOINO JUNIOR, A.; SANTA-CECÍLIA, L.V.C.; SOUZA, G.C. Seleção de isolados de fungos e nematóides entomopatogênicos para a cochonilha-da-raiz-do-cafeeiro Dysmicoccus texensis (Tinsley). Arquivos do Instituto Biológico, v.71, p.181-187, 2004.

BATISTA FILHO, A.; ALVES, L.F.A.; MUNIZ, J.P. Determinação da eficiência de três concentrações de Beauveria bassiana (Bals.) Vuill. no controle de Hypothenemus hampei (Ferrari, 1867) (Coleoptera: Scolytidae). Revista de Agricultura, v.67, p.167-170, 1992. BOUCIAS, D.G.; PENDLAND, J.C. General properties of fungal pathogens. In: BOUCIAS, D.G.; PENDLAND, J.C. Principles of insects pathology. Boston: Kluwer Academic Publishers, 1998. p.259-283.

BURGES, D.H. Formulation of mycoinsecticides. In: BURGES, D.H. Formulation of microbial biopesticides: beneficial microorganisms, nematodes and seed treatments. Boston: Kluwer Academic Publishers, 1998. p.133-185.
CHARNLEY, A.K. Entomopathogenic fungi and their role in pest control. In: WICKLOW, D.; SODERSTROM, M. (Ed.). The mycota IV: environmental and microbial relationships. Heidelberg: SpringerVerlag, 1997. p.185-201.

FERNANDES, P.M.; LECUONA, R.E.; ALVES, S.B. Patogenicidade de Beauveria bassiana (Bals.) Vuill. à broca-do-café, Hypothenemus hampei (Ferrari, 1867) (Coleoptera: Scolytidae). Ecossistema, v.10, p.176-181, 1985.

FERNANDEZ, S.; GRODEN, E.; VANDENBERG, J.D.; FURLONG, M.J. The effect of mode of exposure to Beauveria bassiana on conidia acquisition and host mortality of Colorado potato beetle, Leptinotarsa decemlineata. Journal of Invertebrate Pathology, v.77, p.217-226, 2001.

GONZÁLEZ GARCÍA, M.T.; POSADA FLOREZ, F.J.; BUSTILLO PARDEY, A.E. Desarrollo de un bioensayo para evaluar la patogenicidad de Beauveria bassiana sobre Hypothenemus hampei. Cenicafé, v.44, p.93-102, 1993.

HADDAD, M. de L. Utilização do Polo-PC para análise de Probit. In: ALVES, S.B. Controle microbiano de insetos. Piracicaba: Fealq, 1998. p.999-1013.

IGNOFFO, C.M.; McINTOSH, A.H.; GARCIA, C.; KROHA, M.; JONSON, J.M. Effects of sucessive in vitro and in vivo passages on the virulence of the entomopathogenic fungus, Nomuraea rileyi. Entomophaga, v.27, p.371-378, 1982.

LEITE, L.G.; BATISTA FILHO, A.; ALMEIDA, J.E.M. de; ALVES, S.B. Produção de fungos entomopatogênicos. Piracicaba: Esalq, 2003. 92p.

LeORA SOFTWARE. Poloplus 1.0 probit and logit analysis. Berkeley, 2003. 1 CD-ROM.

LOUREIRO, E. de S.; MONTEIRO, A.C. Patogenicidade de isolados de três fungos entomopatogênicos a soldados de Atta sexdens sexdens (Linnaeus, 1758) (Hymenoptera: formicidae). Revista Árvore, v.29, p.553-561, 2005.

NAKANO, O.; SILVEIRA NETO, S.; ZUCCHI, R.A. Entomologia econômica. Piracicaba: Esalq, 1981.314p.

NEVES, P.M.O.J.; HIROSE, E. Seleção de isolados de Beauveria bassiana para o controle biológico da broca-do-café, Hypothenemus hampei (Ferrari) (Coleoptera: Scolytidae). Neotropical Entomology, v.34, p.77-82, 2005.

ROBERTSON, J.L.; SMITH, K.C.; SAVIN, N.E.; LAVIGNE, R.J. Effects of dose selection and sample-size on the precision of lethal dose estimates in dose mortality regression. Journal of Economic Entomology, v.77, p.833-837, 1984.

ROHDE, C.; ALVES, L.F.A.; NEVES, P.M.O.J.; ALVES, S.B.; SILVA, E.R.L. da; ALMEIDA, J.E.M. de. Seleção de isolados de Beauveria bassiana (Bals.) Vuill. e Metarhizium anisopliae (Metsch.) Sorok. contra o cascudinho Alphitobius diaperinus (Panzer) (Coleoptera: Tenebrionidae). Neotropical Entomology, v.35, p.231-240, 2006.

VÉLEZ ARANGO, P.E.; POSADA FLOREZ, F.J.; MARÍN DE GRISALES, P.; GONZÁLEZ GARCÍA, M.T.; OSORIO VALENCIA, E.; BUSTILLO PARDEY, A.E. Técnicas para el control de calidad de formulaciones de hongos entomopatógenos. Chinchina: Federación Nacional de Cafeteros de Colombia/Cenicafé, 1997. 37p. (Cenicafé. Boletín técnico, 17). 